\title{
Transport Characteristics of Molecular Motors *
}

\author{
Lukasz Machura *,1, Marcin Kostur, Jerzy Łuczka \\ Institute of Physics, University of Silesia, 40-007 Katowice, Poland
}

\section{Abstract}

Properties of transport of molecular motors are investigated. A simplified model based on the concept of Brownian ratchets is applied. We analyze a stochastic equation of motion by means of numerical methods. The transport is systematically studied with respect to its energetic efficiency and quality expressed by an effective diffusion coefficient. We demonstrate the role of friction and non-equilibrium driving on the transport quantifiers and identify regions of a parameter space where motors are optimally transported.

Key words: transport, molecular motors, diffusion, efficiency, Brownian dynamics PACS: 05.60.Cd, 05.40. a, 05.45. a

\section{Introduction}

In the process of evolution Nature has created perfect biomachines. Researchers (read: visionaries) have dreamed to get ahead of Nature and construct molecular-level machines which would be at the ultimate limit of miniaturization. Recent development of nanofabrication technology establishes a foundation for designing, synthesizing, constructing and testing functional hybrid mechanical and electrical devices on a nanometre scale. Such nanomachines, operating under different conditions, could find very many new applications. Nowadays, there are many problems that have to be overcome: how to power such systems, actuate and transport them, and couple to another systems. Nature prompts and provides partial solutions: Within every living cell there is a service structure for its effective function and operation, transport and distribution of various products and substances inside, outside and across cells (Howard, 2001). These processes are mediated by tiny biomotors that move along filamentous tracks. They do mechanical work consuming energy taken from the hydrolization of adenosine triphosphate (ATP) which operates as a power station. The superfamilies of molecular bio-motors like kinesin, dynein and myosin are responsible for trans-

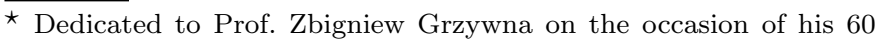
birthday. Presented at the ZJG60 Symposium on Bionanomaterials with Structure-Property Relationship, 10-11 March, Gliwice, Poland * Corresponding author.

Email address: Lukasz.Machura@us.edu.pl (Lukasz Machura).

1 Supported by the Foundation for Polish Science (FNP)
}

port of vesicles and organelles, chromosome segregation, cell division and muscle contraction, to mention only a few. Although biological, chemical and mechanical aspects have been studied, relatively little is known about the mechanisms how the biological motors work. The fundamental question is whether their movement is purely deterministic or rather random which only looks like deterministic on a macro-scale.

Biological motors possess many of the characteristics required to power molecular machines. They can generate force and torque, transport various cargoes and are able to operate in a processive manner, i. e. they can move continuously along the specific substrates for distances of up to hundreds of steps (several microns). An example of such a motor is conventional kinesin (Howard, 2001). The two heads of the kinesin dimer work in a coordinated manner to move along one of 13 protofilament tracks of the microtubule. Each protofilament consists of asymmetric $\alpha \beta$-tubulin heterodimers. A heterodimer is about $8 \mathrm{~nm}$ long and is asymmetric because it is composed of two globular subunits: $\alpha$-tubulin and $\beta$-tubulin which are joined together in a head-to-tail fashion so that the dimmer has a translational symmetry. Because the $\alpha \beta$-tubulin heterodimers are asymmetrical, the microtubule is polar and its ends are structurally different. One consequence of this polarity is that polymerization is faster at one end than the other. The fast-growing end is called the plus-end, whereas the slow-growing end is called the minus-end. The conventional kinesin moves towards the plus-end. There are other bio-motors like e.g. ncd dimer (Henningsen and 
Schliwa, 1997; Sablin et al., 1998) which move in the opposite direction on the same structure. The reason, why the motor moves in a given direction, is not explained to the end. There are several conjectures supported by analysis of very simple mechanical models. One of them says that it can depend on size of the motor, another says that it can depend on mass of the motor or more enigmatically it depends on "molecular topology of the motor domain relative to the rest of the molecule" (Henningsen and Schliwa, 1997).

Artificial molecular motors are under constructions and experiments on transport properties are performed. An example is a Brownian motor by using cold atoms in a dissipative optical lattice. In experiments the optical potential is spatially symmetric and the time symmetry of the system is broken by applying appropriate zero-mean ac forces. As a result, a current of atoms through the optical lattice can be generated (Jones at al., 2004; Sjolund et al., 2006).

\section{Model}

From a physical point of view, the kinesin (or other biomotors) moves in a one dimensional spatially periodic potential $V(x)=V(x+L)$ of period $L \approx 8 n m$ (Visscher et al., 1999) . The reflection symmetry of this potential is broken $V(x) \neq V(-x)$, because the ab-heterodimers, that build the microtubule, a highway for the biomachine, are asymmetrical. This is a crucial fact because it determines the mechanism of the kinesin movement - the ratchet effect (Astumian and Hänggi, 2002; Hänggi et al., 2005; Łuczka et al., 1995). To explain this mechanism, let us consider as simple model as possible. We assume that the molecular motor is a particle of mass $m$ moving in a periodic potential $V(x)$ of period L and of the barrier height $\Delta V=V_{\max }-$ $V_{\text {min }}$. The equation of motion for the motor is the Newton equation with a complementary random force which corresponds to thermal fluctuations, i.e. the Langevin equation in the form

$m \ddot{x}+\gamma \dot{x}=f(x)+g(t)+\sqrt{2 D} \Gamma(t)$

Inertial effects, related to the mass, are described by the first term on the left hand side. The dissipation is included via the Stokes force with the friction coefficient $\gamma$ which is proportional to linear size $R$ of the particle i.e.,

$\gamma=6 \pi \eta R$

and is additionally determined by the viscosity $\eta$ of the medium the particle moves in. The potential force

$f(x)=-\frac{d V(x)}{d x}$

is zero over a period $\mathrm{L}$,

$\langle f(x)\rangle_{L}=\frac{1}{L} \int_{0}^{L} f(x) d x=\frac{1}{L}[V(L)-V(0)]=0$.
The stochastic force $\Gamma(t)$ describes thermal fluctuations which can be modeled by $\delta$-correlated Gaussian white noise of the statistics

$\langle\Gamma(t)\rangle=0, \quad\langle\Gamma(t) \Gamma(s)\rangle=\delta(t-s)$.

According to the dissipation-fluctuation theorem, the thermal noise intensity $D$ is related to the friction coefficient and temperature $T$ of the system, i.e.,

$D=\gamma k_{B} T$,

where $k_{B}$ stands for the Boltzmann constant.

The external time-dependent force $g(t)$ can be of any genre, both deterministic or stochastic (Luczka, 1999; Kula et al., 1998). This force makes the system to be in a nonequilibrium state and is a source of energy for movement of motors. For biological motors it comes from chemical reactions. Here, as an example and for the didactic purposes, we choose a time periodic force, namely

$g(t)=A \cos (\Omega t)$,

where $A$ is the amplitude and $\Omega$ stands for the frequency of the external stimulus. Equivalently we can define the period $T=2 \pi / \Omega$ of the external force. This kind of force can be realized for artificial motors while for biological motors, random non-equilibrium force is more adequate.

\subsection{Time scales}

In physics only relations between scales of length, time and energy are relevant, not their absolute values. Therefore we shell now translate the above equation of motion into its dimensionless form. First of all we determine characteristic quantities - time and length. The characteristic length is the period $\mathrm{L}$ of the potential $V(x)$ and accordingly the coordinate of the molecular motor can be scaled as

$y=\frac{x}{L}$.

Time can be scaled in several ways. One of the possibilities is the relaxation time $\tau_{L}$ of velocity or the correlation time of the velocity of the massive Brownian particle moving freely and driven only by the thermal noise. It can be extracted from a special case of Eq. (1) when the right hand side is zero, i.e.,

$m \dot{v}+\gamma v=0$.

From the above equation it follows

$v(t)=v(0) \exp \left(-t / \tau_{L}\right)$,

where the characteristic time $\tau_{L}=m / \gamma$ is sometimes called the Langevin time. Another characteristic time comes from the overdamped motion of the particle in the potential $V(x)$, when Eq. (1) reduces to the form

$\gamma \frac{d x}{d t}=-\frac{d V(x)}{d x}$. 
Then, by inserting into above equation the characteristic quantities, we get the definition of time $\tau_{0}$,

$\gamma \frac{L}{\tau_{0}}=\frac{\Delta V}{L}, \quad \rightarrow \quad \tau_{0}=\frac{\gamma L^{2}}{\Delta V}$.

During the time interval $\tau_{0}$ overdamped particle proceeds the distance $L$ under the influence of the constant force $\Delta V / L$. Third characteristic time follows from the friction less equation of motion, i.e., when Eq. (1) takes the form

$m \frac{d^{2} x}{d t^{2}}=-\frac{d V(x)}{d x}$.

From the above equation the characteristic time $\tau_{m}$ is given by the relations

$m \frac{L}{\tau_{m}^{2}}=\frac{\Delta V}{L}, \quad \rightarrow \quad \tau_{m}^{2}=\frac{m L^{2}}{\Delta V}$.

One can distinguish also other characteristic times like time period of the external driving $T$ or the well known Einstein diffusion time

$\tau_{E}=\frac{L^{2}}{2 D_{E}}, \quad D_{E}=\frac{k_{B} T}{\gamma}$.

Now we can rescale the equation of motion for the massive Brownian particle in several ways. Doing this we shell take as the relevant time scales these times which differs for different systems, like $\tau_{m}$ or $\tau_{0}$. Let us note that the Langevin time and the Einstein diffusion time do not depend on the system itself, i.e., on the potential and the external driving forces.

\subsection{Rescaled equations of motion}

Let us first propose $t_{0}$ as the relevant time scale, i.e. the rescaled dimensionless time is $s=t / \tau_{0}$. The dimensionless form of the Langevin equation then reads

$\varepsilon \ddot{y}(s)+\dot{y}(s)=F(y)+G(s)+\sqrt{2 D_{0}} \xi(s)$,

where dot denotes derivative with respect to the rescaled time $s, F(y)=-d W(y) / d y=-W^{\prime}(y)$ denotes the rescaled potential force and $G(s)=(L / \Delta V) g(t)=a \cos (\omega s)$ stands for the rescaled external driving force with the rescaled amplitude $a=(L / \Delta V) A$ and frequency $\omega=\Omega \tau_{0}$. The rescaled spatially periodic potential $W(y)=V(x) / \Delta V=$ $V(L y) / \Delta V=W(y+1)$ has the unit period and the unit barrier height. The dimensionless mass

$\varepsilon=\frac{m}{\gamma \tau_{0}}=\frac{\tau_{L}}{\tau_{0}}$

is a ratio of the two characteristic times. The rescaled thermal noise $\xi(s)=(L / \Delta V) \Gamma(t)=(L / \Delta V) \Gamma\left(\tau_{0} s\right)$ has exactly the same statistical properties as $\Gamma(t)$. The dimensionless noise intensity

$D_{0}=\frac{k_{B} T}{\Delta V}$ is a ratio of thermal energy to activation energy the particle needs to traverse the non-rescaled potential barrier.

On the other hand, if we choose the dimensionless time $u=t / \tau_{m}$ and $\tau_{m}$ as a time scale then we end up with another version of the rescaled Langevin equation, namely

$\ddot{y}(u)+\hat{\gamma} \dot{y}(u)=F(y)+G(u)+\sqrt{2 \hat{\gamma} D_{0}} \xi(u)$.

The dimensionless friction coefficient is a ratio of two characteristic times, different then previous two, namely,

$\hat{\gamma}=\gamma \frac{\tau_{m}}{m}=\frac{\tau_{m}}{\tau_{L}}$.

The rescaled driving force $G(u)=a \cos (\omega u)$ with the rescaled amplitude $a=(L / \Delta V) A$ and frequency $\omega=\Omega \tau_{m}$.

Two different scaling are useful in two limiting regimes: Eq. (16) in the overdamped case (when $\varepsilon<<1$ is a small parameter) while Eq. (19) in the underdamped case (when $\hat{\gamma}<<1$ is a small parameter).

\subsection{Numerical values for kinesin}

Let us evaluate characteristic times for one of the best known biological motor, namely, kinesin which moves along a microtubule. As already mentioned in the Introduction, microtubules are spatially periodic structures of period $L \approx$ $8 \mathrm{~nm}$. The mass of the kinesin head domain is of order $m=$ $100 \mathrm{kDa}=1.66 \cdot 10^{-22} \mathrm{~kg}$ and its radius is $R=3 \mathrm{~nm}$. The friction coefficient $\gamma=6 \cdot 10^{-11} \mathrm{~kg} / \mathrm{s}$ is calculated from the Stokes formula with the use of the viscosity of water $\left(\eta=10^{-3} \mathrm{~kg} / \mathrm{ms}\right)$. In a typical Brownian domain the activation energy is 5 time higher than the thermal energy, $\Delta V=5 k_{B} T$ and the temperature inside cell is about $310 \mathrm{~K}$ $\left(37^{\circ} \mathrm{C}\right)$. W can now estimate the typical characteristic times for the kinesin moving inside human cell; thus

$$
\begin{aligned}
\tau_{L} & =2.77 \cdot 10^{-12} s, \quad \tau_{0}=1.8 \cdot 10^{-7} s, \\
\tau_{m} & =7 \cdot 10^{-10} s, \quad \tau_{E}=4.57 \cdot 10^{-7} s .
\end{aligned}
$$

The dimensionless mass (17) in the first scaling and the dimensionless friction (20) in the second scaling have the following values

$\varepsilon=1.54 \cdot 10^{-5} \ll 1, \quad \gamma=2.5 \cdot 10^{2}$.

One can note that the value of the parameter $\varepsilon$ is very small. Therefore Eq. (16) seems to be more appropriate than Eq. (19) because (16) contains the small parameter $\varepsilon$. This allows, with a very good approximation, to put formally $\varepsilon=0$ in the dimensionless equation of motion (16) yielding

$\dot{y}(s)=F(y)+G(s)+\sqrt{2 D_{0}} \xi(s)$.

Analysis of this equation is much easier than Eq. (16). From (21) it follows that the case of overdamped dynamics takes place for biological motors. On the other hand, Eq. (19) contains the parameter $\hat{\gamma}$ and one cannot find justifiable arguments to neglect any term in it. So, this equation is 
much harder to analyze in a complete manner. However, if one wants to investigate some particular effects like the influence of the inertia on the transport, Eq. (19) is more practicable. For other motors, especially non-biological, inertial effect can be crucial. An example is an atomic Brownian motor moving in optical lattices (Brown and Renzoni, 2008) and Eq. (1) in (Hagman et al., 2008). It is worth to stress that if the non-equilibrium driving is of the form (7), the dynamics can be chaotic in some regimes leading to anomalous transport behavior like negative mobility or negative conductivity (Machura et al., 2007; Kostur et al., 2008). Below, we present characteristics of transport in full regime, from underdamped to overdamped one. From now on we will use the later scaling (19) while defining performance characteristics of the motion of the Brownian motor and shell omit all the hats in Eq. (19), for the sake of simplicity.

\section{Performance characteristics of molecular motor}

When one study the motion of molecular motors, the most important transport measure is an average stationary velocity $\langle v\rangle$ of the motor (Machura et al., 2004). Averaging should be performed over all realization of thermal noise, initial conditions and over a period of the external driving. Average velocity describes how much time a typical particle needs to overcome a given distance in the asymptotic (long-time) state. This average velocity, however, is not the only transport attribute. Other characteristics are also important. We will analyze two following transport aspects: quality and energetic efficiency. The quality of transport can be characterized by the effective diffusion coefficient $D_{\text {eff }}$, i.e., by the fluctuations in the position space (Lindner et al., 2001)

$D_{e f f}=\frac{\left\langle x^{2}\right\rangle-\langle x\rangle^{2}}{2 t}$

where the brackets $\langle\ldots\rangle$ denote averaging over all realizations of thermal noise and initial conditions.

The third quantifier is the efficiency of noise rectification (Machura et al., 2004; Suzuki \& Munakata, 2003). The motor moves in viscous media. Therefore the minimal energy input required to move a particle in presence of friction $\gamma$ over a given distance depends on the velocity. In this case, the rectification efficiency is given by the formula (Machura et al., 2005; Suzuki \& Munakata, 2003; Linke et al., 2005; Kostur et al., 2006)

$\eta=\frac{\gamma\langle v\rangle^{2}}{P_{i n}}$

The average input power $P_{i n}$ corresponding to system described by Eq. (19) is given by the formula

$P_{\text {in }}=\gamma\left[\left\langle v^{2}\right\rangle-D_{0}\right]$ where $D_{0}$ is defined in Eq. (18). This expression follows from the energy balance of the underlying equation of motion (19) (Machura et al., 2004).

\section{Numerical experiment and discussion}

To examplify the above ideas we analyze Eq. (19) in the long-time asymptotic, time-periodic regime after effects of the initial conditions and transient processes have died out. Then, the statistical quantifiers of interest can be determined in terms of the statistical average over the different realizations of the process (19) and over the driving period $T$. Since there are no analytical methods to handle (19), we made use of numerical approach and have carried out extensive and precise numerical simulations, applying Stochastic Runge-Kutta algorithm of order 2. For the initial conditions we took points from uniformly distributed circle in the phase space $(x, v)$ with the radius $r=\sqrt{x^{2}+v^{2}}=1$ and the origin in the point $\left(x_{\text {min }}, 0\right)$, where $x_{\text {min }}$ denotes minimum of the potential $W(y)$. For the illustration of the above idea we fixed the following set of parameters: $\omega=$ $4.9, D_{0}=0.001,0.05$ and the potential profile $W(y)=$ $\Delta W[\sin (2 \pi y)+0.25 \sin (4 \pi y)]$, where $\Delta W=0.454$ reduces the maximal barrier height to unity. The forces corresponding to this potential ranges from the minimal value -2.14 to the maximal value 4.28 . If the amplitude $a$ of the driving is higher than 4.28 , the motor is able to overcome the potential barrier in any direction just with the use of the external driving force and without of thermal noise. We analyze the system (19) in the 2-dimensional parameter space $\{\gamma, a\}$. We change the rescaled friction coefficient $\gamma$ from $\gamma=0.001$ (underdamped dynamics) to $\gamma=100$ (overdamped dynamics). The external time periodic force strength $a$ varies between 0 and 5 , where the later is just above the maximal value of the potential force.

In Fig. 1 we show three characteristics in the low temperature regime, $D_{0}=0.001$, cf. Eq. (18). In panel (a), we depict the stationary average velocity of the motor. White area corresponds to the case when the absolute value of the velocity is small, $|\langle v\rangle|<10^{-2}$. Blue (or gray) areas surrounded by contours stands for the negative mean velocity and red (gray) color indicates regions of positive velocity. It is seen that for a chosen set of parameters the Brownian motor has a noticeable velocity for the force amplitude $a>$ 2 and for not strong damping $\gamma<2$. Strictly speaking, the velocity is not exactly zero assuming very small values of order $10^{-2} \div 10^{-7}$ (white area on Fig. 1 (a)). By inspecting the several colored (gray) areas, one can observe that the velocity as a function of $\gamma$ or the amplitude $a$ exhibits the multiple velocity reversals (Kostur and Łuczka, 2001). Because the friction coefficient $\gamma$ depends on the linear size of the motor, it means that motors of different sizes can move in opposite directions. In panel (b) we show the rectification efficiency (23). There, white area corresponds to efficiency smaller that $\eta<10^{-3}$. The correlation of dark regions in panels (a) and (b) is evident. There are two main islands of 


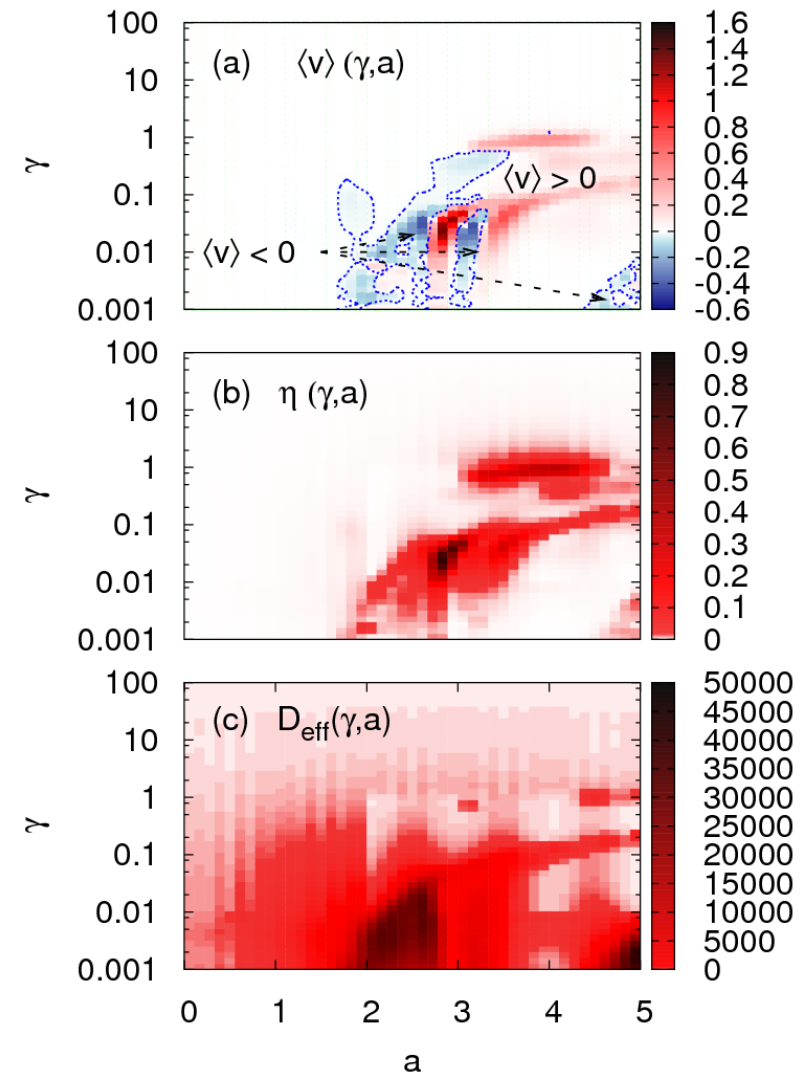

Fig. 1. (color online) Performance characteristics of the molecular motor are presented versus the external force amplitude $a$ and friction coefficient $\gamma$. In panel (a) we show the average velocity $|\langle v\rangle|<10^{-2}$ (white), negative (blue, gray surrounded by contour), positive (red, gray). In panel (b) we present the efficiency. In panel (c) we depict the effective diffusion. Other parameters are: $D_{0}=0.001, \omega=4.9$.

efficient energy conversion - one for $\{\gamma, a\} \in\{0.4 \div 2,3 \div 5\}$ and second for $\{\gamma, a\} \in\{0.001 \div 0.2,2 \div 5\}$. In the region of parameters $\{\gamma \approx 0.002, a \approx 2.9\}$, the motor efficiency is very high and is almost $90 \%$ (remember that the system is far from an equilibrium state). In panel (c) we show the effective diffusion coefficient of the motor. Large value of this quantifier means low quality of motion - Brownian particles move in a very irregular manner. This region of high efficiency and low quality of motion (high diffusion), makes our stochastic model similar to deterministic (Mateos, 2000) or Hamiltonian (Schanz et al., 2001) systems.

Upon the inspection of Fig. 1 one can notice that for the parameter island with large negative average velocity and high efficiency (large area around $\gamma=0.02$ and $a=2.8$ ), the motor moves quite irregularly because $D_{\text {eff }}$ is large. For the island with large positive velocity and medium efficiency (smaller area around $\gamma=1$ and $a=4$ ), the effective diffusion is small reflecting good quality of transport and regular motion.

Let us consider the case of higher temperature, $D_{0}=$ 0.05. Transitions of a particle over potential barriers are easier and details of the shape of the potential is not so important now. As one can see from panel (a) of Fig. 2, absolute values of the average velocity are one order smaller

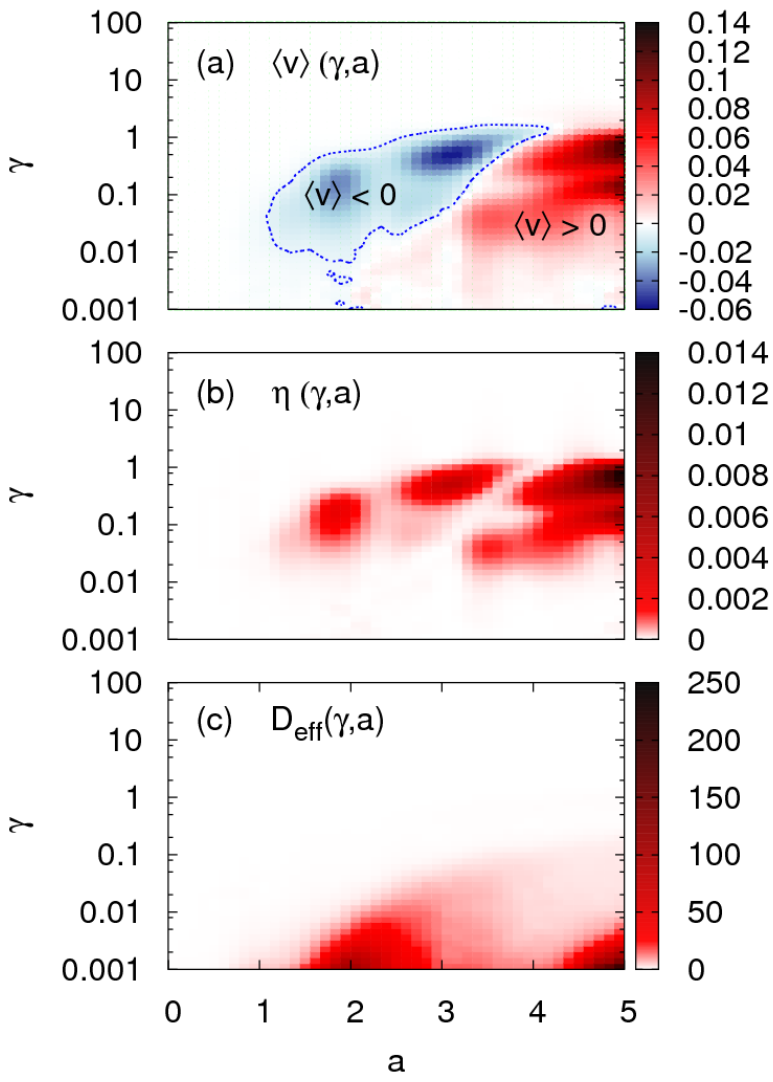

Fig. 2. (color online) The same as in Fig. 1 but for higher temperature, $D_{0}=0.05$. Panel (a): the average velocity $|\langle v\rangle|<10^{-3}$ (white), negative (blue, gray surrounded by contour), positive (red, gray). Panel (b): the efficiency. Panel (c): the effective diffusion.

than in the previous temperature regime. Higher temperature makes the motor motion slower in both negative blue (gray) contoured areas, or positive - red (gray) colored regions. Again as in the previous case, in the the 2dimensional parameter space $\{\gamma, a\}$, we can identify the velocity reversal phenomenon upon the change of the control parameters $a$ or $\gamma$. In the negative direction the maximal velocity $\langle v\rangle=-0.06$ and in the positive direction $\langle v\rangle=0.14$. In the optimal regime, the efficiency of the motor transport is of order $1 \%$. The only characteristic which seems to be better than in the previous scenario is the effective diffusion presented in panel (c). The motor seems to move in a much more regular manner than for lower temperature yielding maximum value of $D_{e f f}=250$ for underdamped system. It is a region where the averaged velocity is almost zero and the efficiency is very low.

Comparison of Figs 1 and 2 leads to the conclusion that the influence of higher temperature is rather destructive. The only better quantifier is the effective diffusion coefficient which is smaller. It means that the islands in the parameter space $\{\gamma, a\}$ for higher temperature with relatively high (negative or positive) velocity accompanied by the highest possible efficiency represent regimes where the motor moves rather in a regular way. 


\section{Conclusions}

The most demanded properties of any transporting machinery are: efficiency and quality. The latter in our case is characterized by the effective diffusion coefficient. Let us note that even in any equilibrium system the particle can be transported over long distances due to thermal diffusion. It is, however, very unreliable when the distance becomes large because the diffusion cannot distinguish direction and most of traveling particles would not arrive at prescribed destination in a reasonable time. On the other hand, from Figs 1 and 2 we can see that the large diffusion is sometimes in regimes where the energetic efficiency is high. In real situations, Nature chooses between above scenarios. Under some circumstances, if e.g. the distances are small, the diffusion can be exploited to transport the cargo and no energy is wasted. When the distance is larger, then the non-equilibrium transport is applied and reliability is achieved sacrificing the energy input.

\section{Acknowledgments}

The work supported in part by the Polish Ministry of Science and Higher Education (Grant No. 202131 32/3786). One of the authors (LM) gratefully acknowledges financial support by the Foundation for Polish Science (FNP).

\section{References}

Astumian R. D., Hänggi, P., 2002. Brownian motors. Phys.Today 55, 33-39.

Brown M. and Renzoni F.,2008. Ratchet effect in an optical lattice with biharmonic driving: A numerical analysis. Phys. Rev. A 77, 033405.

Hagman H., Dion C. M., Sjlund P., Petra S. J. H., Kastberg A., 2008. Influence of the lattice topography on a three-dimensional, controllable Brownian motor. Europhys. Lett. 81, 33001.

Hänggi, P., Marchesoni, F., Nori, F., 2005. Brownian motors. Ann. Phys. (Leipzig) 14, 51-70.

Henningsen U., Schliwa M., (1997). Reversal in the direction of movement of a molecular motor. Nature 389, 9395.

Howard, J., 2001. Mechanics of Motor Proteins and the Cytoskeleton. Sinauer Associates Sunderland.

P. H. Jones, M. Goonasekera, and F. Renzoni, Rectifying Fluctuations in an Optical Lattice. Phys. Rev. Lett. 93, 073904 (2004).

Kostur, M., and Łuczka, J., 2001. Mutiple current reversals in Brownian motors. Phys. Rev. E 63, 021101.

Kostur, M., Machura, L., Hänggi, P., Łuczka, J., Talkner, P., 2006. Forcing inertial Brownian motors: Efficiency and negative differential mobility. Physica A 371, 20-24.

Kostur M., Machura L., Talkner P., Hänggi P., Łuczka J., 2008. Anomalous transport in biased ac-driven Joseph- son junctions: Negative conductances. Phys. Rev. B 77, 104509.

Kula J, Kostur M, Łuczka J, 1998. Brownian transport controlled by dichotomic and thermal fluctuations. Chem. Phys. 235, 27-37.

Landau, L. D., Lifshitz. E. M., 1959. Fluid Dynamics. Pergamon Press, Oxford.

Lindner, B., Kostur, M., Schimansky-Geier, L., 2001. Optimal Diffusive Transport in a Tilted Periodic Potential. Fluct. Noise Lett 1, R25-R40.

Linke, H., Downton, M. T., Zuckermann, M. J., 2005. Performance characteristic of Brownian motors. Chaos 15, 026111.

Luczka, J., 1999. Application of statistical mechanics to stochastic transport. Physica A 274, 200-215.

Łuczka, J., Bartussek, R., Hänggi, P., 1995. White Noise Induced Transport in Periodic Structures. Europhys. Lett. 31, 431-436.

Machura, L., Kostur, M., Talkner, P., Łuczka, J., Marchesoni, F., Hänggi, P., 2004. Brownian motors: current fluctuations and rectification efficiency. Phys. Rev. E 70, 061105 .

Machura, L., Kostur, M., Marchesoni, F., Talkner, P., Hänggi, P., Łuczka, J., 2005. Optimal strategy for controlling transport in inertial Brownian motors. J. Phys.: Condens. Matter 17, S3741-S3752.

Machura, L., Kostur, M., Marchesoni, F., Talkner, P., Hänggi, P., Łuczka, J., 2006. Addendum and Erratum: Optimal strategy for controlling transport in inertial Brownian motors. J. Phys.: Condens. Matter 18, 41114112.

Machura L., Kostur M., Talkner P., Łuczka J., Hänggi P., 2007. Absolute negative mobility induced by thermal equilibrium fluctuations. Phys. Rev. Lett. 98, 040601.

Mateos, J. L., 2000. Chaotic Transport and Current Reversal in Deterministic Ratchets, Phys. Rev. Lett. 84, 258 261.

Peclet, E., 1843. Traite de la Chaleur Consideree dans ses Applications. Hachette, Paris.

Sablin E. P., Case R. B., Dai S. C., Hart C. L., Ruby A., Vale R. D., Fletterick R. J., 1998. Direction determination in the minus-end-directed kinesin motor ncd. Nature 395, 813-816.

Schanz H., Otto M.-F., Ketzmerick R., Dittrich T., 2001. Classical and quantum Hamiltonian ratchets. Phys. Rev. Lett. 87, 070601.

Sjolund P., Petra S. J. H., Dion C. M., Jonsell S., Nylen M., Sanchez-Palencia L., Kastberg A., 2006. Demonstration of a Controllable Three-Dimensional Brownian Motor in Symmetric Potentials. Phys. Rev. Lett. 96, 190602.

Suzuki, D., Munakata, T., 2003. Rectification efficiency of a Brownian motor. Phys. Rev. E. 68, 021906.

Visscher, K., Schnitzer, M. J., Block, S. M., 1999. Single kinesin molecules studied with a molecular force clamp. Nature 400 184-189. 